\title{
RECOMBINATION IN THE AM GENE OF NEUROSPORA CRASSA-A NEW MODEL FOR CONVERSION POLARITY AND AN EXPLANATION FOR A MARKER EFFECT
}

\author{
J. R. S. FINCHAM \\ Department of Genetics, University of Leeds, Leeds LS2 $9 \mathrm{JT}$
}

Received 13.ix.75

\begin{abstract}
SUMmary
It is argued that polarised intragenic recombination is not necessarily due to hybrid DNA extending into the gene for variable distances from one side; it can as well be explained by hybrid DNA usually covering the whole gene, the two complementary DNA strands of the gene having unequal chances of undergoing the interchromatid transfer involved in hybrid DNA formation, and excision from base-pair mismatches proceeding predominantly in one chemical direction (perhaps $5^{\prime}$ to $3^{\prime}$ ). This alternative model for polarity is thought to be in better accord with the behaviour of flanking markers in the am data, which appears to show that conversion-associated crossing-over can occur with almost equal likelihood on either side of the gene (a feature previously held to support negative interference-Fincham, 1974). It also provides an explanation for an unusual marker effect involving two am mutational sites separated by only three base pairs. The nature of the sites (defined by amino acid replacement analysis) is such that one forms a purine-purine mismatch on the same chromatid as the other forms a pyrimidine-pyrimidine mismatch, and vice versa. They show an approximately two-fold difference in recombination frequencies in crosses to other am mutants mapping to the left, and a difference of similar magnitude, but opposite sign, in crosses to mutants mapping to the right.
\end{abstract}

\section{INTRODUGTION}

THE am gene of Neurospora crassa codes for NADP-linked glutamate dehydrogenase, a protein with a polypeptide chain 452 amino acid residues in length. The complete sequence of the chain has recently been published (Holder et al., 1975) and the amino acid replacements brought about by several mutations have been defined (G. K. Chambers, A. A. Holder and M. Brett, unpublished).

Attempts to map sites of mutation within the am gene have been made both by Fincham (1967) and by Smyth (1973). In both studies the approach was the conventional one of making crosses between single auxotrophic mutants, selecting and counting prototrophic recombinants and classifying these recombinants with respect to flanking markers placed on each side of the gene. The basic type of cross may be represented as follows:

\begin{tabular}{llll}
$P$ & $l$ & + & $D$ \\
\hline$p$ & + & $r$ & $d$ \\
\hline
\end{tabular}

where $P / p$ and $D / d$ are proximal and distal flanking markers respectively and $l$ and $r$ are "left" and "right" mutant sites within the gene. In such studies in fungi, ordering on the basis of prototroph frequencies is not usually very convincing, and more reliance is placed on the relative frequencies, 
among the selected prototrophs, of the parental $(P D$ and $p d)$ and recombinant $(P d$ and $p D)$ flanking marker arrangements.

In spite of some differences between the respective experimental designsthe use of different distal flanking markers and the presence in all Smyth's stocks of $r e c-3$, a recessive increasing recombination within am by about tenfold-the results of the two studies were qualitatively very similar. One striking result was the relatively low degree of association of intragenic recombination and crossing-over of flanking markers; the $P d$ and $p D$ classes together amounted generally to only some 25 to 35 per cent of the $\mathrm{am}^{+}$ recombinants. A similarly low probability of crossing-over accompanying intragenic recombination has been reported for several other genes in Neurospora (e.g. Murray, 1963; Smith, 1965), and it is apparent that the 50 per cent association which holds (at least approximately) in yeast (Hurst et al., 1972) is better regarded as an upper limit than as a general rule. This paper will be more concerned with two other features of the am data which present greater difficulties of interpretation. These are:

(i) A consistent tendency for $P D$ to outnumber $p d$ among the $a m^{+}$recombinants by three or four to one.

(ii) A much smaller inequality, or an approximate equality, of $P d$ and $p D$.

Observation (ii) means that the conventional criterion for site order, namely the relationship $p D>P d$, is not very useful in this case. On the other hand, observation (i) does permit the $a m$ sites to be put in a consistent linear order which, at least so far, is co-linear with the order of the corresponding amino acid replacements in the gene product (G. K. Chambers, A. A. Holder and M. Brett, unpublished). One can rationalise the success of observation (i) in this regard by postulating a polarity in gene conversion, such that the left hand site in any particular cross is converted from mutant to wild more frequently that is the right hand site. The model for polarised conversion which has usually been favoured states: (a) that recombination within the gene depends on conversion occurring at one site and not the other (since co-conversion does not give recombinants but only converts one mutant into the other); (b) conversion at a given site depends on that site being present in hybrid DNA without the other, since, within one stretch of hybrid DNA co-conversion is the rule; (c) hybrid DNA tends to extend into the gene from some point to one side of it (in our convention, to the left). This explanation, while adequate for observation (i) above, leads to complications in relation to observation (ii), at least if one adheres to the general recombination model of Holliday (1968) or the very plausible and attractive derivative of it recently proposed by Meselson and Radding (1975). According to these models, the crossing-over associated with conversion should occur immediately at one end of the hybrid DNA; if hybrid DNA formation is polarised to one side of the gene the conversion-associated cross-overs should occur predominantly on that side, and there should be as great and consistent an inequality of $p D$ and $P d$ as there is between $P D$ and $p d$. The nearequality of the two recombinant flanking marker arrangements might, in itself, suggest that the hybrid DNA responsible for the intragenic recombination tended, most of the time, to cover both of the heterozygous sites within the gene, but to postulate this would be to abandon the usual explanation of conversion polarity. In a previous discussion of this dilemma (Fincham, 
1974), I suggested that most of the conversion-associated cross-overs should be regarded as correlated but separate events, not attributed to the same region of hybrid DNA as caused the intragenic event. This was, in effect, to revive the idea of negative interference, or clustered recombinational events (Pritchard, 1960).

It is the purpose of this paper to show that an alternative way of accounting for the data is to adopt an entirely different view of the mechanism of polarity in conversion. On what scem to be quite realistic assumptions one can, in fact, expect to find apparent conversion polarity even in cases where hybrid DNA tends to extend right across the gene. The model to be proposed is also capable for accounting for a strong and unusual marker effect which is shown both in my data and in Smyth's, but which has not previously been discussed.

\section{A NEW MODEL FOR CONVERSION POLARITY}

I propose that the hybrid DNA which provides the opportunity for recombination within am normally covers all or most of the gene, and that there need be no polarity as regards the direction from which it enters the gene. In this situation an apparent conversion polarity (i.e. a consistent inequality of the $P D$ and $p d$ classes of $a m^{+}$recombinants) will follow provided that two conditions are fulfilled:

(i) It is always, or at least usually, a DNA strand of a particular polarity which undergoes inter-chromatid transfer to form the hybrid DNA. This condition seems by no means unreasonable, since it is very likely that the single-strand nicks, which must be necessary for strand exchange, are generated by an endonuclease which can discriminate between different sequences, perhaps acting at special recombination " hotspots".

(ii) Conversion (i.e. base mismatch correction) is initiated by an endonucleolytic incision at or close to a mismatched base-pair, followed by singlestrand degradation through one of the mismatched nucleotides and extending beyond it in one direction. For the sake of argument, and by analogy with the bacterial system for the excision of UV-induced pyrimidine dimers (Kushner et al., 1971) we may suppose that this direction is $5^{\prime}$ to $3^{\prime}$.

The postulated situation is shown diagrammatically in fig. 1. For convenience in the presentation of the argument the figure shows reciprocal exchange of DNA single strands between chromatids, but it makes no difference to the conclusions if the transfer is non-reciprocal-the two reciprocally constituted hybrid duplexes would then occur with statistically equal frequencies in different cells rather than paired in the same cell.

Since the experimental system is to select only for prototrophic recombinants, we need only consider the cases where it is the mutant member of a mismatched base pair which is excised. It will be seen from fig. 1 that, if excision is unidirectional, the two chromatids marked respectively with $P D$ and $p d$ are in quite different situations with regard to excision of mutant bases. In the $P D$ chromatid (in the convention of the figure) excision from the mutant members of each of the two mismatched sites will be divergent, while in the $p d$ chromatid it will be convergent. In the former case, degradation and repair synthesis can bring about mutant-to-wild conversion at the two sites without mutual interference. In the latter case, the outcome will depend on the length of the excised tract of DNA in relation to the inter-site 
distance, and the degree of synchrony of the events at the two sites. If synchrony is not perfect, and if the excision tract is generally longer than the inter-site distance, then conversion initiated at one site will pre-empt conversion at the other-that is to say, one excision tract will run across both sites and the result will be co-conversion without recombination. Thus most $a m^{+}$ recombinants will be formed on the $P D$ rather than the $p d$ chromatid. In
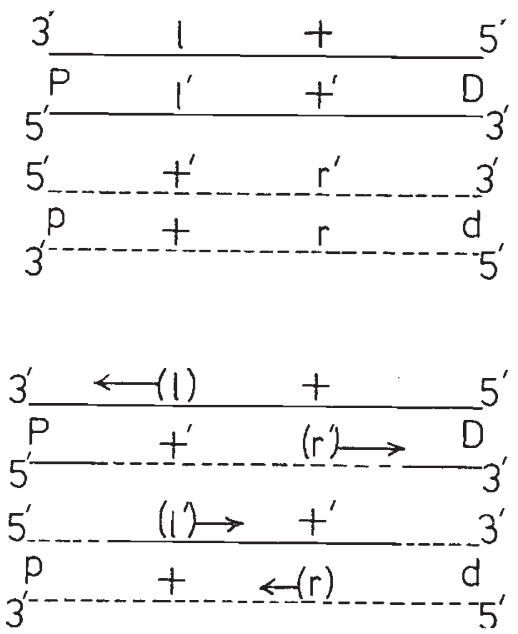

FIG. 1.-The proposed model for polarised intragenic recombination. The upper part of the fig. shows the two parental chromatids and the lower part the same two chromatids after exchange of segments of single DNA strands of like polarity, covering the gene. $P / p$ and $D / d$ are proximal and distal flanking markers respectively; $l$ and $r$ are "left " and " right" mutant sites within the gene, with their wild-type equivalents represented by + , and primed and unprimed symbols indicating complementary bases. The brackets indicate excision of a mismatched base and the arrows show the preferred direction of single-strand degradation.

general, if the non-exchanged DNA strand undergoes leftwards degradation, it will be the parental chromatid carrying the left-most mutant site which will yield most of the wild type recombinants. The $P D: p d$ inequality will define left-right relationships within the gene irrespective of which particular pair of mutants supply the "left" and " right" sites in any particular cross. On this model the apparent conversion polarity reflects the unequal involvement of the two complementary DNA single strands in interchromatid transfer; there is no gradient, either in probability of hybrid DNA formation or in probability of mismatch correction once hybrid DNA is formed, over the whole gene. It should also be noted that there is nothing in the situation as described which enables one to correlate "left" and " right" within the gene with "proximal" and "distal" as defined by the flanking markers.

\section{A POLARISED TWO-WAY MARKER EFFEGT}

Although the map of the am sites based on recombination frequencies is not as clear as that based on the $P D: p d$ inequality, there evidently is some relationship between recombination frequency and inter-site distance. Indeed, Pateman (appendix to Fincham, 1967) deduced an order close to that obtained from the polarity criterion from recombination frequencies 
alone. One major anomaly in the frequencies concerns sites 2 and 19, which are now known (G. K. Chambers and A. A. Holder, unpublished), to affect adjacent residues in the polypeptide chain. In $a m^{19}, \mathrm{Lys}^{141}$ is replaced by Met, while in $a m^{2}$, His ${ }^{142}$ is replaced by Gln. In terms of codons, $a m^{19}$ is

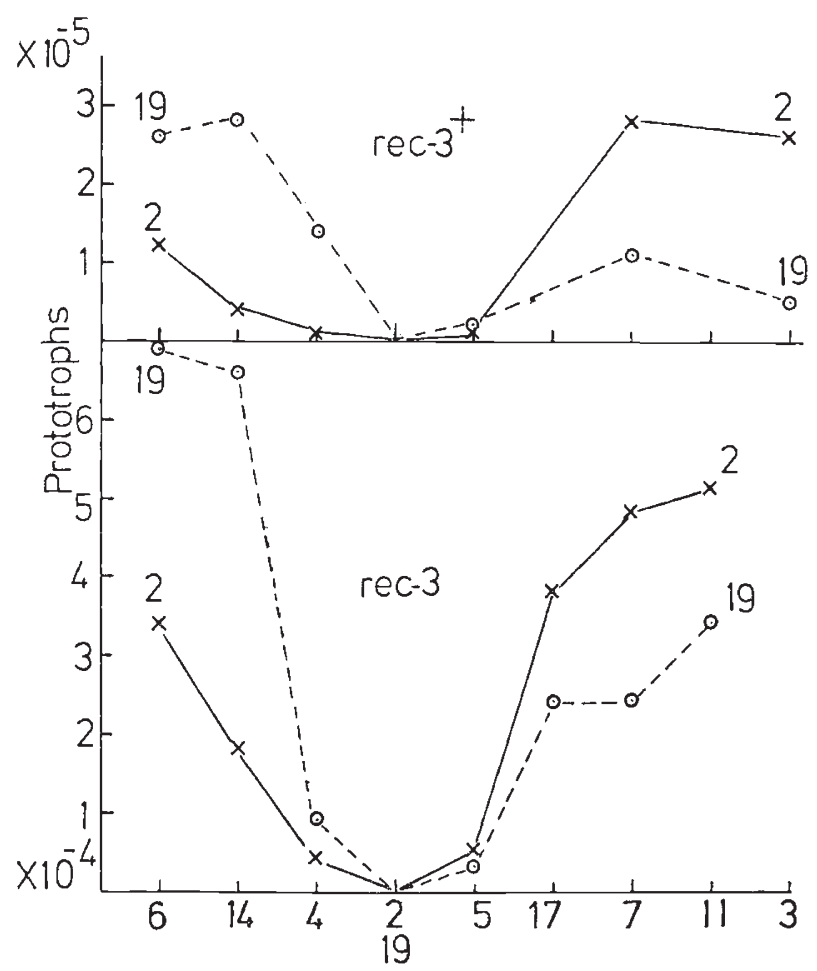

Fig. 2.-The recombination (prototroph) frequencies shown by $a m_{2}$ and $a m_{19}$ in crosses with other mutants in the am gene. The mutant sites are indicated in what is thought to be their correct order, but spaced at arbitrarily equal distances. The upper and lower parts of the fig., based on data from Fincham (1967) and Smyth (1971) respectively, show the effect on recombination frequencies of the rec-3/rec-3+ difference.

changed from AAG to AUG and $a m^{2}$ from CAPy to GAPu (where Py represent either pyrimidine and $\mathrm{Pu}$ either purine). The base-pair substitutions in the two mutants are separated by only three other base-pairs, and it is not surprising to find that they show extremely little recombination with each other $\left(0.05 \times 10^{-5}\right.$ prototrophs in the rec-3 background as compared with about $100 \times 10^{-5}$ between the ends of the gene map-D. G. Catcheside, personal communication). However, the two mutants show very different frequencies in crosses with other mutants within the gene and, even more unexpectedly, the sign of the difference is reversed as one passes from sites to the left to sites to the right. In crosses to sites to the left, 19 gives two or more times as many recombinants as 2 , while in crosses to sites to the right 2 gives 1.5 or more times as many as 19 . The difference is (with the sole exception of crosses to $a m^{5}$ in the rec- $3^{+}$background where the number of recombinants counted was excessively small) completely consistent in both sets of data, and it extends over three sites to the left and five to the right. The data are shown in fig. 2. 
The finding of a marker effect both to the left and to the right of sites 2 and 19 is unexpected on the basis of a model for conversion polarity in which (since it is always the leftmost site which is preferentially converted) most recombinants arose from cells in which hybrid DNA covered the left site only. While a difference in the efficiency of mismatch correction between sites 2 and 19 could affect frequencies of recombination in crosses to sites to

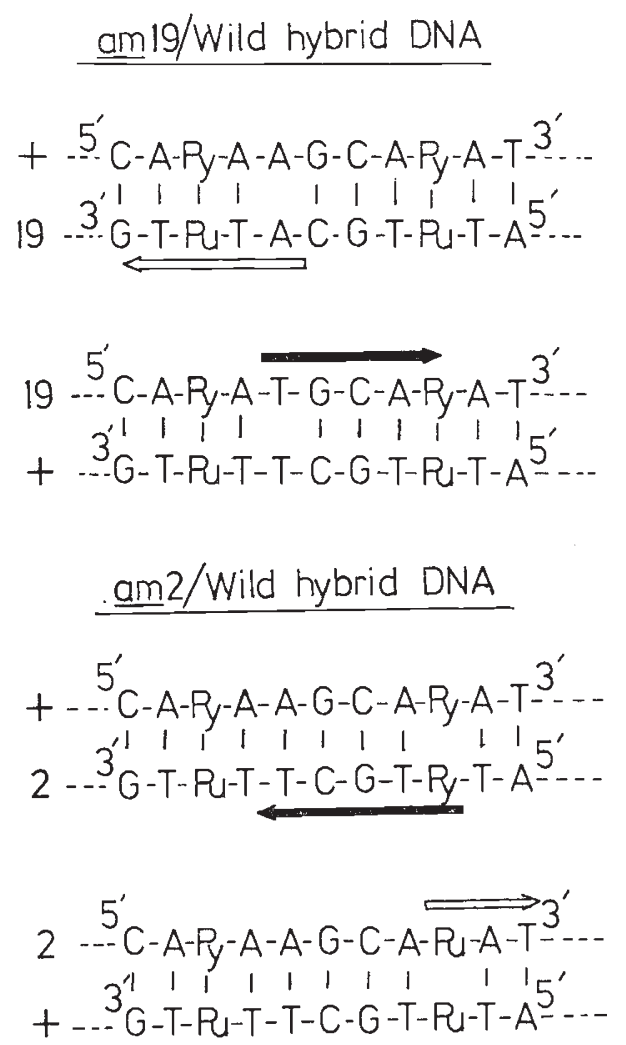

FIG. 3.-The nature and positions of mutant/wild base-pair mismatches in the $a m_{2}-a m_{19}$ region of the am gene. The arrows indicate the postulated preferred direction of singlestrand degradation, and the solid and open arrows indicate high efficiency and low efficiency excision respectively, on the assumption that an endonuclease acts preferentially at pyrimidine-pyrimidine mismatches. Pu stands for any purine and Py for any pyrimidine, subject to Pu-Py pairs being complementary.

the right, one would not expect, on this hypothesis, much difference from this cause in crosses with sites to the left.

The alternative model for conversion polarity outlined in the preceding section can, however, explain the two-way marker effect; indeed, it almost predicts it. Exchange of single DNA strands in the vicinity of sites 2 and 19 will produce the situations shown in fig. 3 . Heterozygosity with respect to $a m^{2}$ will result in a pyrimidine-pyrimidine mismatch in the upper chromatid as drawn in the figure and a purine-purine mismatch in the lower one. Heterozygosity with respect to $a m^{19}$ will give the opposite result-a purine-purine (adenine-adenine) mismatch in the upper chromatid and a pyrimidine- 
pyrimidine (thymine-thymine) mismatch in the lower one. It seems very likely that any endonuclease recognising mismatches in hybrid DNA will discriminate between these alternatives. Suppose, for the sake of argument, that it is the pyrimidine-pyrimidine mismatches which are recognised most efficiently. Then in crosses heterozygous for $\mathrm{am}^{2}$ it will be the upper chromatid (as drawn in the figure) which will have the greatest chance of correction from mutant to wild type, assuming that degradation proceeds in the $5^{\prime}$ to $3^{\prime}$ direction; in crosses heterozygous for $a m^{19}$ the favoured chromatid will be the lower one. In the case of $a m^{2}$, excision in the favoured chromatid will proceed to the left, while in the case of $a m^{19}$ it will proceed to the right. Hence, following the argument of the preceding section that most recombinants result from divergent rather than convergent correction tracts, $a m^{19}$ will re-combine more efficiently with sites to the left and $a m^{2}$ with sites to the right, which is the result observed. It is, of course, possible to turn the whole argument upside-down and postulate that the correction endonuclease prefers to recognise purine mismatches and that excision proceeds $3^{\prime}$ to $5^{\prime}$.

\section{Implications AND Limitations OF THE MODEL}

\section{(i) Relationship between recombination frequency and distance}

The most serious difficulty arising from the model as just described is that it fails to explain the relationship which evidently exists between recombination frequency and inter-site distance. If most recombinants within a gene were formed as a result of divergent excision as shown in figs. 1 and 3, one would expect recombination frequency to be nearly independent of distance down to very close spacings of sites. In fact, there is no doubt that, in general, intragenic recombination frequencies extrapolate (in approximately linear fashion) to zero at zero distance, and it was for this reason that unidirectional excision was ruled out in favour of bidirectional excision in the explanation of map expansion given by Fincham and Holliday (1970). On the present model, a limitation on recombinant yield would indeed be encountered at extremely close spacings if the endonucleolytic nick provoked by the base-pair mismatch was apt to occur several base-pairs away from the mismatch on its $5^{\prime}$ side, prior to $5^{\prime}$ to $3^{\prime}$ degradation. If this were the case, then, with sites only a few base-pairs apart, the potentially divergent excision tracts would actually overlap both sites, and co-conversion would occur to the exclusion of recombination even on the upper chromatid depicted in the lower part of fig. 1. It seems very unlikely, however, that this can be the whole explanation of the frequency-distance relationship because of the comparatively distant spacings of sites at which restriction of recombination becomes apparent. For example, in the am gene, site 7, which maps between 1 and 3, is separated from each of these latter sites by as little as 2-3 per cent of the gene map, in terms of recombination frequency. The amino acid replacements in $a m^{1}$ and $a m^{3}$ are now known (A. A. Holder and M. Brett, unpublished) and they show that sites 1 and 3 are 171 base-pairs apart. To explain restriction of recombination frequency over distances of this magnitude it seems necessary to abandon the idea of exclusively unidirectional excision and to allow that, while the relevant nuclease is most effective in one direction it, or another enzyme, can enlarge the gap (albeit to a lesser and variable extent) on the other side also. While this rather ad hoc elabo- 
ration detracts from the simplicity and elegance of the model, it seems plausible from the biochemical point of view and consistent with all the genetic information. The general explanation for map expansion developed by Fincham and Holliday (1970) remains tenable on this basis.

\section{(ii) The initiation of strand exchange}

To explain the whole pattern of recombination in the am gene we need one more special assumption for this particular case. In terms of the model, the $P D>p d$ relationship in the $a m^{+}$recombinants implies that it is predominantly DNA strands of one polarity which are exchanged between chromatids. On the other hand, the near-equality of $P d$ and $p D$ Suggests that the hybrid DNA can be initiated on either side of the gene without very much bias. The nicks which free the DNA strands for hybridisation should therefore occur in at least two places, one on each side of the gene. It will be no more than a coincidence if these two vulnerable sequences happen to occur on the same strand rather than on strands of opposite polarity. Such a coincidence would not be unlikely-it would, in fact, occur 50 per cent of the time, other things being equal-but there is no reason why it should be a general rule.

\section{(iii) Consequences in tetrads}

The predictions of the new model for tetrad data will not be set out in detail here, but are easily seen from a consideration of fig. 1. Very different predictions are made depending on whether exchange of DNA single strands between chromatids is reciprocal or non-reciprocal.

If strand exchange is reciprocal, then, even though divergent correction will generate wild type $(++)$ recombinants exclusively on the $P D$ chromatid, a $3: 1$ wild to mutant ratio can occur either at site $l$ or at site $r$ depending on whether convergent correction on the $p d$ chromatid restores $+r$ or brings about co-conversion to $l+$. The other possibility, divergent correction on both chromatids, will give wild-type and double-mutant recombinants in the same tetrad-that is the appearance of "reciprocal crossing-over" within the gene. The $P D>p d$ relationship in wild-type recombinants (and conversely, the $p d>P D$ relationship in double-mutant recombinants) will serve to define the left-right relationships of sites, but there will be no conversion polarity in the sense of one site showing 3:1 ratios in preference to the other.

If, on the other hand, single-strand transfer is non-reciprocal, with restoration of the original genotype of the donor chromatid by repair synthesis (as in the model of Meselson and Radding, 1975), a ++ recombinant generated by divergent correction on the $P D$ chromatid will always be accompanied in the same tetrad by $+r$ on the $p d$ chromatid-that is, the $3: 1$ ratio will always be at site $l$. Furthermore, apparent reciprocal crossovers within the gene will not occur. Thus, given the assumption of nonreciprocal transfer, the present model is capable of explaining the classical "polaron" effect in tetrads first described for Ascobolus immersus by Lissouba et al. (1962). There is, however, an important proviso to be made. The proposed explanation of polarity requires that co-conversion should be frequent. In fact, on simplest assumptions, one can show that co-conversion covering sites $l$ and $r$ together should be 50 per cent more frequent than 
conversion at $l$ alone. The classical polarons of Ascobolus immersus were detected in octads selected as containing wild-type recombinants, and the frequencies of co-conversion were not determined. Some relevant data from yeast, where total analysis of unselected tetrads did detect co-conversions, argues against the present model. In the yeast gene arg-4, the data of Hurst et al. (1972) show a consistent conversion polarity extending across six sites. Five of these sites do show reasonably high frequencies of co-conversion, but the left-most site undergoes conversion largely independently of the others while still continuing the same gradient of conversion frequency (in fact, it shows the lowest frequency in two-point crosses). A case such as this suggests that the older explanation of polarity, involving hybrid DNA spreading into the gene from one side for a variable distance, must still be retained, at least as one possibility.

It is not the purpose of this paper to argue for one mechanism of conversion polarity to the exclusion of the other; it is rather to point out that at least two mechanisms are possible and that, on quite reasonable assumptions, we may expect both to apply in different cases. Indeed, the complex patterns of recombination seen within some genes may reflect the operation of both effects simultaneously.

Acknowledgments. - I thank Dr R. Holliday for comments on a draft of this paper, and Professor D. G. Catcheside for communicating to me his unpublished recombination data on $a m_{2} \times a m_{19}$ crosses.

\section{REFERENCES}

FINCham, J. R. s. 1967. Recombination within the am gene of Neurospora crassa. Genet. Res., Camb., 9, 49-62.

FInCham, J. R. S. 1974. Negative interference and the use of flanking markers in finestructure genetic analysis. Heredity, 33, 116-121.

FINCHAM, J. R. S., AND HOLLIDAY, R. 1970. An explanation of fine structure map expansion in terms of excision repair. Molec. Gen. Genet., 109, 309-322.

holder, A. A., WOotton, J. C., BARon, A. J., Chambers, G. K., AND Fincham, J. R. s. 1975. The amino acid sequence of Neurospora NADP-specific glutamate dehydrogenase. Peptic and chymotryptic peptides and the complete sequence. Biochem. $7 ., 140$.

HOllidAy, R. 1968. Genetic recombination in fungi. In Replication and Recombination of the Genetic Material, eds. W. D. Peacock and R. D. Brock. Austr. Acad. Sci., Canberra.

HURST, D. D., FOGEL, s., AND MORTIMER, R. K. 1972. Conversion-associated recombination in yeast. Proc. natn. Acad. Sci. U.S.A., 69, 101-105.

KUSHNER, S. R., KAPlan, J. C., ONO, H., AND GROSSMAN, L. 1971. Enzymatic repair of deoxyribonucleic acid. IV. Mechanism of photoproduct excision. Biochemistry, 10, 33253342.

LISSOUBA, P., MOUSSEAU, J., RIZET, G., AND ROSSIGNOL, J. L. 1962. Fine structure of genes in the Ascomycete Ascobolus immersus. Adv. Genet., 11, 343-380.

MESELSON, M. S., AND RADDING, C. M. 1975. A general model for genetic recombination. Proc. natn. Acad. Sci. U.S.A., 72, 358-361.

MURRAY, N. E. 1963. Polarized recombination and fine structure within the $m e-2$ gene of Neurospora crassa. Genetics, 48, 1163-1184.

PRITCHARD, R. H. 1960. Localized negative interference and its bearing on models of genetic recombination. Genet. Res., Camb., 1, 1-24.

Sмiтн, B. R. 1965. Interallelic recombination at the histidine-5 locus in Neurospora crassa. Heredity, 20, 257-276.

SмYтH, D. R. 1973. A new map of the amination-1 locus of Neurospora crassa and the effect of the recombination-3 gene. Austr. F. Biol. Sci., 26, 1355-1370. 